\title{
Las dos culturas: Ciencias Naturales y Ciencias Humanas en el siglo ${X X I^{1}}^{1}$
}

\section{"The two cultures: Natural Sciences and Human Sciences in the 21st century"}

Aceptación: marzo 2008.

Dr. Cristián Parker G.

Aprobación: mayo 2008.

\section{RESUMEN}

El articulo examina la concepción que los estudiantes universitarios chilenos tienen acerca de sus representaciones y valoraciones de las ciencias, la tecnologia y la ética. Se sistematizan los principales resultados de las encuestas realizadas entre 2005 y 2008, en el marco de proyectos FONDECYT la primera a una muestra representativa de 6.219 estudiantes de todas las universidades del Consejo de Rectores y la segunda, a una muestra de 421 estudiantes representativa de tres universidades estatales: Universidad de Chile, Universidad de Concepción y Universidad de Santiago de Chile, se interroga precisamente acerca del grado en que está presente, en la mentalidad estudiantil, la distinción entre ciencias lógico-experimenta. les y arte-humanidades. Se entregan algunos resultados preliminares acerca de la forma de semantización de ciencia y tecnologia en estudiantes de carreras en los polos extremos: carreras tecnológicas, por una parte, y carreras de humanidades, por otra.

Palabras clave: Mentalidad universitarios, concepciones de la ciencia, tecnologia, ciencias experimentales, humanidades.

\begin{abstract}
The article examines the conceptions that Chilean university students have about their representation and valuation of the sciences, technology and ethics. It presents a systematization of the principal results of interviews done between 2005 and 2008 in the framework of FONDECYT projects. The first was a representative sample of 6,219 students from all the universities of the Council of Rectors and the second a sample of 421 students representative of three state universities: Universidad de Chile, Universidad de Concepción and Universidad de Santiago de Chile. The survey seeks to discover to what degree precisely the distinction between logical-experimental sciences and arts-humanities is present in student mentality. Some preliminary results are presented about the way in which science and technology is semanticized by students in courses at opposite extremes: technology courses on the one hand and humanities courses on the other.
\end{abstract}

Key words: University student mentality, conceptions of science, technology, experimental sciences, humanities.

Este trabajo se ha desarrollado en el marco del proyecto FONDECYT N ${ }^{\circ} 1070172$.

Doctor en Sociologia, Director del Instituto de Estudios Avanzados, Universidad de Santiago de Chite, cparker@usach.cl

DOI: $10.7770 /$ CUHSO-V16N2-ART277 
Revista CUHSO volumen $16 \mathrm{~N}^{\circ} 2$

\section{Las dos culturas}

A fines de los años 50 el científico y literato Charles Percy Snow, publicó una famosa obra titulada The Two Cultures and the Scientific Revolution (1959) en la que hacia notar que el quiebre y falta de comunicación entre las humanidades y las ciencias (en el sentido estricto de ciencias experimentales) era uno de los mayores obstáculos para resolver los problemas del mundo contemporáneo. Se quejaba de la ignorancia de parte de los científicos de las grandes obras del humanismo y de los humanistas que carecian de ciertos conocimientos básicos de las ciencias naturales contemporáneas.

Habermas, retomando el tema, cita un artículo de Aldous Huxley (1979) en que este autor analiza las diferencias y las remite a las diferentes experiencias en que está basada la literatura y las ciencias. Para el hombre de letras "la realidad exterior se relaciona constantemente con el mundo interior de la experiencia privada, la lógica compartida se modula para convertirse en sentimiento no compartido, la salvaje individualidad quiebra siempre la cáscara de la costumbre cultural" (Huxley, 1979: 12); en cambio, el hombre de ciencia se centra en el mundo exterior, públi$\mathrm{co}$, y hace lo posible por ignorar los mundos que le revelan las más privadas experiencias propias y las ajenas.

Según Huxley, el mundo de la literatura es en el cual los hombre nacen, viven y se mueren, tienen alegrías y sufimientos, anhelos y desesperanzas, amores y desamores, estupideces y sabidurías, mundo de contraste entre la razón y la pasión, entre los institintos y las convenciones, entre el lenguaje común y lo inexpresable. El mundo de la ciencia, por su parte, no es un mundo de fenómenos dados, sino de estructuras finas descubiertas, no un mundo de eventos únicos de la experiencia y la multiplicidad de sus matices cualitativos, sino un mundo de regularidades cuantificadas.

Ahora bien debe existir una forma de comunicación entre estos mundos más allá de las relaciones de oposición, subsunción o indiferencia, que ha existido hasta el momento, y Habermas (1973) nos recuerda, difiriendo con
Huxley, que se trata precisamente de que las informaciones propiamente científicas no pueden entrar en contacto con el mundo de la vida social "sino por la puesta en valor técnico, es decir, en tanto que saber tecnológico: y allí ellas (las ciencias) nos sirven para disponer técnicamente de las cosas" (Habermas, 1973: 78).

Habermas intenta resolver el problema por la vía del reconocimiento de la "instrumentalidad" de la ciencia-técnica, pero existe una vía de solución que apunta al fondo del problema y esto es a la visión epistemológica subyacente a la división entre ciencias naturales y ciencias humanas. Nos referiremos a esta cuestión más adeante.

Por otro lado, si bien Habermas no supera una epistemología clásica en cuanto reconoce que la ciencia-tecnología están dominadas por una racionalidad instrumental y pueden ser objeto de un conocimiento "cientifico objetivo", sin embargo por su valoración de la relación entre valor técnico y mundo de la vida, concede un reconocimiento a un estatuto epistémico diferenciado entre el saber cientifico y el saber de la vida cotidiana.

Por ello, esas valoraciones no se sitúan en el mismo plano que las concepciones que se hacen los grupos sociales sobre sí mismos y que orientan su acción. La ideología social y política no se ubica en el mismo plano que las representaciones de la ciencia y la tecnología. Con esta afirmación, dicho sea de paso, Habermas refuta el riesgo del relativismo de las teorias radicalmente constructivistas de las ciencias y las tecnologías que las reducen a meras formas nominalistas de lenguaje experto.

Pero hay que notar, a su vez, que la postura habermasiana tiene sus críticos que objetan una suerte de contaminación sustancialista. En efecto, Feenberg (1999) llega incluso a afirmar que el enfoque de Habermas (1973) es también esencialista en relación a la tecnología dado que la concibe abstractamente como la aplicación de la racionalidad instrumental, cuyo peligro estaría en la contaminación del mundo de la vida y la parálisis de los procesos comunicacionales. 
UNIVERSIDAD CATÓLICA DE TEMUCO

"Desde este punto de vista cualquier intento de infundir significado a lo técnico aparece como una interferencia externa en un campo racional que tiene sus propias leyes y lógica. Aunque pensamiento racional (que lo es), la tecnología engloba a sus creadores, amenazando su supervivencia tanto material como espiritual" (Feenberg, 1999: 2).

El desarrollo de la ciencia y la tecnología es un proceso sociohistórico. La ciencia y la tecnologías estan sometidas a influencias sociohistóricas y son ellas mismas producidas de forma sociohistórica. La ciencia no progresa por simple acumulación de conocimientos, sino que está sometida a una dialéctica según la cual, de vez en cuando, se producen verdaderas "revoluciones" al decir de Khun (2005), en las que los viejos paradigmas son sustituidos por nuevos.

La ciencia y la tecnología no están determinadas (por un mecanicismo unilineal), pero tampoco indeterminadas (por un autonomismo idealista). Son producto de un complejo proceso de sobredeterminación, en el cual confluyen tanto factores propiamente tecno-científicos (autonomía relativa) como factores sociales (determinaciones socio históricas) y ciertamente constituye un lenguaje especializado con significados diversos para el hombre, y sus productos tienen impactos, interferencias y consecuencias para el mundo humano significativo.

II. Estudios sobre la visión de los estudiantes universitarios acerca de ciencias naturales y ciencias humanas

A propóstito de nuestro inquietante tema hemos realizado estudios en estudiantes universitarios acerca de sus representaciones y valoraciones de las ciencias, la tecnología y la ética.
Nos hemos querido interrogar precisamente acerca del grado en que está presente, en la mentalidad estudiantil, la distinción a la que hemos hecho mención: distinción, tradicionalmente conflictiva o problemática, entre ciencias lógico-experimentales y artehumanidades. Y más aún, nos preguntarnos acerca de la forma de semantización de ciencia y tecnología en estudiantes de carreras en los polos extremos: carreras tecnológicas por una parte, y carreras de humanidades, por otra. La interpretación tiene como trasfondo el análisis de los principales resultados de las encuestas realizadas en 2005 y 2008 , en el marco de proyectos FONDECYT ${ }^{3}$. La primera, a una muestra representativa de 6.219 estudiantes de todas las universidades del Consejo de Rectores, y la segunda, a una muestra de 421 estudiantes, representativa de tres de las cuatro universidades más importantes: Universidad de Chile, Universidad de Concepción y Universidad de Santiago de Chile. Aquí nos centraremos, sin embargo, en las metodologias y resultados principalmente cualititativos.

De acuerdo a los principios del método comparativo (Nohlen, 2006), el establecimiento de condiciones para la comparación se da sobre la base de dos casos que comparten rasgos comunes y que en unas cuantas dimensiones se diferencian sustancialmente. De esta manera se podría haber escogido a estudiantes de ciencias biológicas y de ciencias sociales y quizás el abismo entre la visión de las "ciencias" no hubiesre sido tan pronunciado, sobre todo por la proximidad que existe entre algunas ciencias biomédicas y la psicología, por ejemplo. Pero el caso es que hemos tomado los extremos: ingenierias y humanidades, precisamente para fines comparativos.

En efecto, esa conocida polémica entre ciencias naturales y ciencias humanas no solo tiene una tradición intelectual en los clásicos

Investigación FONDECYT 104261 realizada en 2005 sobre base de una encuesta a una muestra representativa de estudiantes universitarios del Consejo de Rectores en Chile. $\mathrm{N}=6219$ e Investigación FONDECYT 1070172 realizada en 2008 sobre base de una encuesta a una muestra representativa de estudiantes de la Universidad de Chile, Universidad de Concepción y Universidad de Santiago de Chile. $\mathrm{N}=421$. Siendo el autor el investigador responsable en ambos investigaciones. Ver Parker 2008a y Parker 2008c. 
sino que también permea una suerte de sentido común de los universitarios: las ciencias serían preferidas por los que estudian carreras científicas y, en cambio, serian criticadas por los humanistas. Asimismo las tecnologías serían preferidas, como indica la intuición, por aquellos estudiantes que precisamente siguen carreras vinculadas a las ingenierías o a las tecnologías.

¿Es efectivamente así? Y si fuese así, ¿qué significa verdaderamente esto que comentamos en términos de la visión que unos y otros estudiantes tienen acerca del significado de la ciencia? Es lo que queremos ilustrar en forma abreviada en este parte de nuestra exposición, para luego respondernos: ¿qué elementos podemos rescatar de estas visiones para nuestra reflexión actual, de cara al siglo XXI, precisamente sobre la relación entre ciencias naturales y ciencias sociales?
En general, los estudiantes universitarios tienen una visión positiva acerca de la ciencia y la tecnología contemporánea. Los estudiantes no son reacios a las ciencias y tampoco se guían por una mentalidad tradicionalista y anticientífica ${ }^{4}$. Las críticas que se hacen a las ciencias provienen más bien desde consideraciones éticas y desde lo que se considera la ambigüedad de las ciencias (y su falibilidad y riesgo) (Parker, 2008a).

La visión acerca de la ciencia y la tecnología varía de acuerdo a las áreas del conocimiento de las carreras en que estudian. En el estudio realizado en el año 2005, el análisis del cruce de estas visiones, de acuerdo a las áreas de conocimiento, evidencia que son precisamente los estudiantes de ciencias exactas y naturales los que tienen la percepción positiva más alta de las ciencias y casi tan alta de la tecnología como los estudiantes de tecno-

Gráfico 1. Visiones positivas de la ciencia y la tecnología por áreas del conocimiento

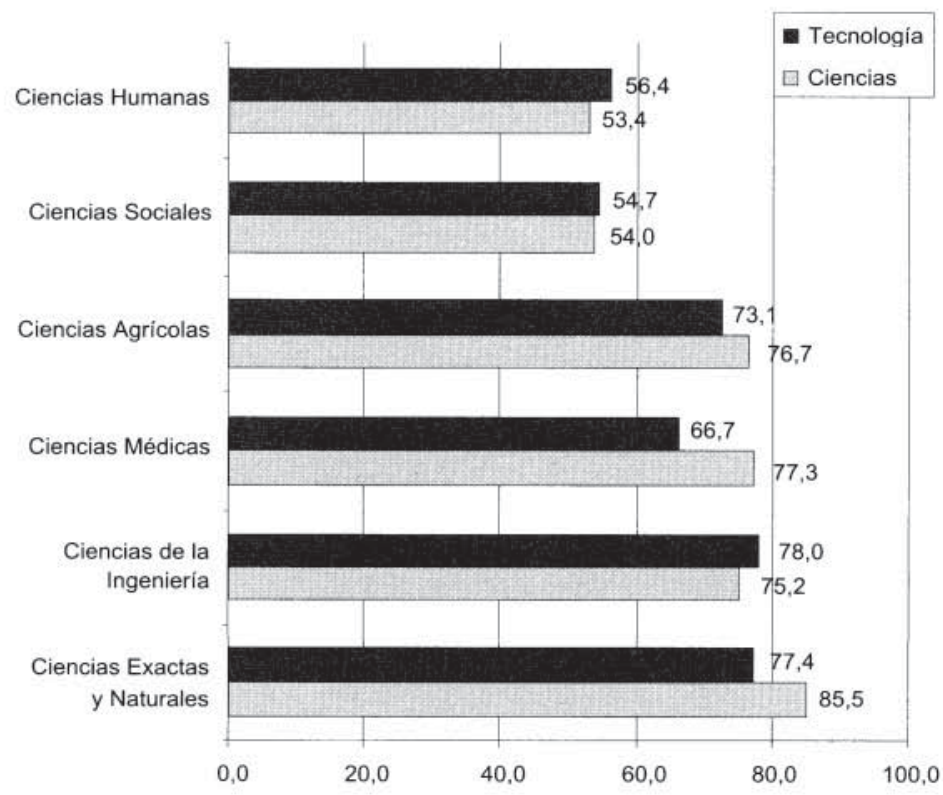

Fuente: Investigación FONDECYT No 1040261; Estudiantes Universitarios 2005; N=6219.

4 En el estudio de 2005 un $67 \%$ de los estudiantes manifiestan una visión bastante positiva de la ciencia y $66 \%$ de ellos manifieste una visión bastante positiva de la tecnología. En general, el análisis conduce a la conclusión de que no existe una mentalidad tradicionalista o anti ciencia en el estudiantado actual. (Parker 2008a y Parker 2008b). 
logías e ingenierias. Le siguen los estudiantes de ciencias médicas y ciencias agrícolas en la valoración positiva de las ciencias (con seguridad debido a la centralidad que tienen las ciencias biológicas en la formación de las carreras universitarias agrupadas bajo esta dos áreas de conocimiento). Los estudiantes que tienen menos valoración de las ciencias son aquellos que sigen carreras inscritas bajo las áreas de las ciencias humanas y sociales.

En cuanto a la tecnología, la más alta valoración de ella proviene de las carreras del área de la ingeniería, luego de ciencias exactas y naturales y, en seguida, de las ciencias agrícolas. La menor valoración positiva proviene de estudiantes de las áreas de ciencias sociales y de las humanidades.

Todo ello estaría conforme a lo razonable, a no ser por un aparente detalle: ¿Qué se entiende por ciencias?, ¿Acaso no está aquí gravitando en el discurso de los estudiantes una acepción reductiva de la "ciencia" asimilándola exclusivamente a las ciencias exactas y naturales? Eso parece estar ocurriendo en la medida en que los alumnos de ciencias sociales indican altas valoraciones negativas de la ciencia e incluso varios de ellos prefieren las tecnologías a las ciencias.

Dada la centralidad de esta encrucijada de miradas, vamos a profundizar en el significado de la ciencia de parte de dos extremos de estudiantes, como hemos dicho: aquellos que se orientan vocacionalmente y siguen carrreas de tecnología/ ingeniería y los que se orientan y siguen carreas de humanidades.

Se realizaron, al efecto, cuatro Focus Groups en grupos selectos de estudiantes de ingeniería (Universidad de Santiago de Chile, USACH, en Santiago, y Universidad de Playa Anacha, UPLA, en Valparaíso) y de estudiantes de humanidades (de las mismas universidades). El análisis de discurso fue realizado con la metodología de la clasificación etnográfica.

Retomamos aquí los resultados fundamentales del análisis en lo que concierne a nuestro tema de fondo: ¿como ven la relación entre humanidades y ciencias (experimentales) los estudiantes universitarios de hoy?
III. Discursos estudiantiles sobre el concepto de ciencia y temas afines

Los estudiantes de humanidades no tienen un claro concepto de ciencias y se refieren al concepto como dotado de "ambiguedad". Se asocia semánticamente la palabra "ciencia" a tecnología. Estudiantes de humanidades de la UPLA afirman que su carrera también tiene que ver con ciencias porque "tiene un ámbito de ciencia, piensa en la información, en todo lo que se refiere a las tecnologías, siempre nos han recalcado el rol que tendriamos que cumplir nosotros en cuanto a las ciencias de la información, el manejo de las nuevas tecnologías, enseñarle a las personas cómo manejarlas, a buscar información y manejarse en todo ámbito de temas"....

Pero el concepto aparece también vinculado con el campo semántico del método, como concepto nuclear, instalado al centro de cualquier forma particular de ciencia, a la vez que como forma de estudio, análisis o acercamiento a una realidad o un objeto.

En general, se dice también que en su conceptualización hay cierta confusión, sobre todo en los medios masivos de comunicación, que tienden a encapsular a las ciencias en el área de la tecnología, existiendo otras áreas de la ciencia como las políticas, sociales y humanas.

Por tanto hay en este discurso un forma de reivindicación de las ciencias humanas y un llamado a no restringir el concepto solo a las ciencias más "duras", ya que "hay que entender que la ciencia tiene muchos rasgos más, como las ciencias humanas, que es la que más interesa"... (Grupo Humanidades, UPLA)

También se entiende que las ciencias están condicionadas culturalmente y que sus paradigmas están sujetos a influencias ideológicas.

"Creo que es una cuestión más bien cultural, debido a que las ciencias se basan en ideas, las ciencias físicas en un momento se basaron en teorías mas mecanicistas, más duras... pasó el tiempo y los avances, las mismas ciencias se dieron cuenta que eso estaba inválido" (Grupo de Humanidades, UPLA). 
Se destacan las diferencias entre ciencias naturales y ciencias humanas.

"Para explicar ciertos fenómenos ya sean naturales, o sean espirituales o de cualquier tipo de indole, social, cultural y se da cierta separación de las ciencias porque hay un minuto en que no se puede explicar de manera fáctica ciertas problemáticas"... (Grupo de Humanidades, UPLA).

Pero se destaca también la importancia actual de los enfoques "interdisciplinarios" y el carácter sociocultural de la propia ciencia natural:

"... una tendencia que está in crescendo en todas las ciencias, la interdisciplinariedad... de repente los profesores marcaban mucho la diferencia entre lo cualitativo y lo cuantitativo e incluso entre las ciencias duras y las blandas, o sea, había dos tendencias que uno tenía el tema social y el otro no, que había un tema de desarrollo metodológico... es la base de la ciencia, el método científico y en las disciplinas de las ciencias sociales utilizan el método científico, lo que pasa es que también llegan a generar a veces campos experimentales, a perder el control, como dices tú, de las variables, pero esa cualidad digamos que se otorgaba a la ciencia física y que podía lograr esos espacios no contaminados... se ha ido avanzando en que la verdad es que siempre están intervenidos, o sea, al crear ese espacio experimental, también genera una intervención humana..." (Grupo de Humanidades, UPLA).

Se describe el rol de la ciencia en base a su aporte al progeso humano, pero se está consciente de que el desarrollo de la ciencia involucra opciones valóricas y no tiene carácter infalible. Muchas veces la ciencia aparece como equívoca y no confiable. También se cuestiona la orientación del progreso que busca la ciencia dado que es necesario reconocer que ella "limita con los valores" y con las condicionantes sociales de la distribución de riquezas que condiciona su acceso.

"... en realidad no sé si la ciencia sea tan asi (benefeciosa), porque están buscan- do la cura pa'l cáncer, pa'l sida, pero en realidad tení que gastar miles de dólares para comprarte el tratamiento, entonces, no se a qué niveles de mejoramiento de la vida social estamos llegando" (Grupo de Humanidades, USACH).

En general, sin embargo, el balance acerca de los beneficios de la ciencia es apreciado por los estudiantes de humanidades como positivo. Los discursos le adscriben a la ciencia un rol preponderante para el desarrollo del país y de la sociedad. No obstante, se tiene la percepción de que el país y sus instituciones no han valorado a la ciencia como una verdadera herramienta de progreso.

Para los estudiantes de ingenierías, en cambio, el concepto de ciencia se relaciona con los descubrimientos, con la acumulación de conocimientos integrados y la rigurosidad científica. Las ciencias son las ciencias naturales y formales:

"La matemática es una ciencia, la ciencia tiene que ver con los descubrimientos, el hombre estudia la ciencia, trata de buscar el porqué de las cosas, en biología, quimica, matemáticas, esas son ciencias..." (Grupo de Ingeniería, UPLA).

Por otra parte, la ciencia aparece como la dimensión más relevante de la cultura humana y tiene que ver con el descrubrimiento de verdades y de sentido:

"La ciencia es todo el conocimiento que el hombre ha logrado obtener a través de todos estos años, gracias a ello nos diferenciamos de las otras especies"... (Grupo de Ingeniería, USACH).

"Creo que más que descubrimientos trata por qué estamos acá, el porqué de las cosas, ciencia es como todo lo que ve al futuro"... (Grupo de Ingeniería, UPLA)

Se caracteriza por su método y la exactitud de sus conocimientos y descubrimientos que son inagotables:

"... todo lo que se ha descubierto, porque siempre va haber algo que descubrir, 
siempre va haber algo que estudiar, más cosas, nuevas carreras, por eso siempre va tener distintos significados y puntos de vista" (Grupo de Ingenieria, UPLA).

"Uno siempre anda buscando cosas para llevarlas al plano de la exactitud, la ciencia es algo que no se va acabar nunca"....

"Ciencia... es como capturar fenómenos de la naturaleza en números y aplicar la rigurosidad científica" (Grupo de Ingeniería, USACH).

Se reconoce la existencia de ciencias naturales y ciencias humanas, se reconoce una diferencia de mentalidad, pero muchos estudiantes de ingeniería valoran el hecho de que últimamente las ciencias sociales y humanas aplican un método científico:

"Hay una diferencia, las ciencias exactas te llevan a algo que puedes ver, las otras te llevan a una opinión, una reflexión que puede cambiar de persona en persona. Si, hay una diferencia en la mentalidad que toma uno al estudiar ciencias" (Grupo de Ingeniería, UPLA).

"Las ciencias sociales no tienen la metodología de las ciencias exactas, no tienen esa rigurosidad, No, yo creo que últimamente se han puesto bien serias las ciencias sociales y han aplicado el método cientifico más rigurosamente" (Grupo de Ingeniería, USACH).

"(Ciencia)... es un estudio, pero un estudio metódico, que busca respuestas y busca resultados y se divide también en ciencias duras, que es donde nos enfocamos nosotros, y en ciencias sociales.... que es un estudio para interpretar fenómenos del hombre" (Grupo de Ingeniería, USACH).

Pero esta diferencia de las dos culturas no solo proviene del método, sino que también estos estudiantes reconocen un cambio en la perspectiva espistemológica que acepta que hayan fenómenos de realidad no explicables con el método científico clásico y no por ello escapan de ser estudiados por una regla sistemática:
"Considero a la ciencia como el conocimiento, pero no solo a las ciencias exactas que buscan interpretar la naturaleza, también creo que las ciencias humanistas son importantes, que ven como otro ámbito, aunque no sean (...) es interpretar la naturaleza pero muchas veces la naturaleza no tiene explicaciones exactas, como no todo tiene su explicación con números, hay muchas ciencias que son prácticamente impredecibles o simplemente existen pero no se pueden demostrar las leyes. (Grupo de Ingeniería, USACH).

Aunque las ciencias humanas y sociales no son suficientemente rigurosas, se abocan al estudio de fenómenos de una mayor complejidad:

"Pero es que son cosas que no son medibles, entonces son, pueden tener hartas interpretaciones, no como las ciencias exactas, no son cosas tan tangibles. Es que las ciencias sociales, claro, tienen muchas más variables que a lo mejor son más dificiles de modelar y de cuantificar, pero si para el estudio de las ciencias humanas se está aplicando el método cientifico" (Grupo de Ingeniería, USACH).

En este sentido se tiene la percepción de que en las aulas de las carreras universitarias de tecnologías o ciencias naturales existe la tendencia a disminuir la importancia y rigurosidad de las ciencias "blandas". Algo que para algunos, tiene que ver con el modelo de sociedad en que se esta inserto, es decir, con formas de reproducción cultural desde la escuela: circula el estigma de que "el 'flojo' estudia ramos humanistas; y el 'aplicado' estudia ciencias 'duras'".

En algunos discursos estudiantiles de carreras tecnológicas se llega a cuestionar la sobreespecialización en su formación:

"Se especializa demasiado el conocimiento, en vez de integrarlo, de hecho, en carreras de ingeniería hay muchos ramos que se han eliminado y que son super importantes para el desarrollo integral del profesional... nosotros vemos pura matemática y no tenemos ningún ramo social, siento un vacío en esa parte"... (Grupo de Ingeniería, USACH). 


\section{Revista CUHSO volumen $16 \mathrm{~N}^{\circ} 2$}

Esto, sin duda se reproduce en las aulas de enseñanza, y tiene muchas veces como consecuencias la no integración entre alumnos de distintas disciplinas, que tiendan a segregarse.

Finalmente estos estudiantes también reconocen el condicionamiento cultural de las ciencias y de las tecnologías.

"... lo he visto, en el campo se usa una tecnología na' que ver, atrasada... tenen otras necesidades, entonces no significa que tengan que aplicar la misma tecnología que tenemos que aplicar nosotros en la ciudad... en el fondo depende del contexto, o sea, no es que sea mejor tecnología o peor tecnología... en países distintos, por ejemplo en países orientales, hoy día mismo en África... tienen que ver también con el contexto de cómo desarrolla el hombre su propia tecnología". (Grupo de Ingeniería, USACH).

IV. Posibilidades y/o amenazas del desarroIlo científico y tecnológico

En cuanto a la visión que los estudiantes universitarios tienen acerca del desarrollo científico-tecnológico futuro, los alumnos de humanidades son más criticos ya que aprecian que éste podria estar afectando e incluso desestructurando la identidad propia y la identidad cultural; estaría afectando las relaciones sociales y deshumanizando la convivencia. El discurso estudiantil tiene variadas afirmaciones en relación a la ciencia y la tecnología con el poder. Muchas veces la tecnología sería transformada en un instrumento de poder al servicio de las potencias o de las grandes empresas. Esto obliga a preguntarse: ¿es el desarrollo científico-tecnológico sinónimo de desarrollo humano?

"... estamos llegando a un punto en que el hombre ya no tiene una identidad propia, estamos llegando mucho hacia la comodidad... ¿hacia dónde queremos ir con el desarrollo? El desarrollo no es malo, ¿cachái?, tampoco es bueno. La tecnología no es buena ni es mala, ahi está, hay que saber utilizarla, con qué fines, si solo vamos a lograr la comodidad, nos vamos $a$ ir a la cresta, ¿cachái? Vamos a llegar a ser personas robotizadas, personas piedras, que no van a tener ni siquiera necesidad de contacto con los demás, y desde ese punto de vista, claro, que es un amenaza" (Grupo de Humanidades, UPLA).

Como dice el párrafo anterior, se aprecia un riesgo de cosificación del hombre en la tecnología. La idea de la alienación tecnológica la expresó otro estudiante del mismo grupo con la frase: "las tecnologías del siglo XXI son una amenaza en la medida que ellas se apropien de nosotros, y no nosotros de ellas, porque en el fondo somos nosotros los que tenemos que apropiarnos de ellas...".

Otro impacto negativo del desarrollo científico-tecnológico está representado por su relación con el medio ambiente. Los insumos para la producción de muchos bienes son recursos naturales no renovables, to que significa la explotación de bosques, minerales y otros recursos en pos de "objetivos tecnológicos", afirma el discurso analizado. Tanto en el análisis del discurso como en una amplia encuesta que fue aplicada a estudiantes de las universidades chilenas, se observa una preocupación marcada por el medio ambiente, una mirada holística y ecológica acerca de cómo concebir al desarrollo humano con una dimensión sustentable (Parker, 2008a).

El desarrollo científico-tecnológico parece ser necesario pero no está excento de graves riesgos que involucran la sustentabilidad futura:

"Es complicado tomar partido por uno o por otro lado, porque como se ha vis. to para todos es útil, todos dependemos de... pero también uno ve las noticias y 7 millones de hectáreas al año se van perdiendo de árboles, de bosque nativo...; como el agujero de la capa de ozono..., como todo eso nos va afectando y va deteriorando el mundo en el cual vivimos, $y$ uno puede decir: no me va a pasar nada, puede que no me pase, pero también ¿como tan inconsciente del mundo que le estamos dejando a la gente que va a venir después?, ¿en que mundo van a vivir...?" (Grupo de Humanidades, USACH). 
Pero no solo el impacto negativo en el medio ambiente trae consigo el desarrollo científico-tecnológico, también el discurso estudiantil de humanidades resalta otro eje temático: la incertidumbre sobre la equidad y el futuro de la humanidad. Esto se relaciona con la problemática social, la desigualdad económica y social en los países y entre ellos, es decir, con las brechas de todo tipo que parecen agudizarse cada día más.

¿Cuál es la igualdad y la democratización que han promovido al pais las nuevas tecnologias? Entonces... no es real lo que nos venden los medios de comunicación... Se tiende a la concentración de las tecnologias, igual que la concentración del poder igual que la concentración de la riqueza sigue quedando en manos de unos pocos, y esto, claro, de repente llegará algún desarrollo tecnológico a lo mejor, está llegando desarrollo tecnológico, pero las cifras siguen alarmantes. El otro día veiamos en estadistica que si el mundo fueran 100 personas, uno tendría computador solamente y 80 nunca hubiesen hablado por teléfono en su vida" (Grupo de Humanidades, USACH).

En los estudiantes de ingenieria, en cambio, el desarrollo científico y tecnológico representa más oportunidades que amenazas. En el ámbito de las oportunidades y beneficios que trae consigo la ciencia y la tecnologia, resttan temáticas de salud, energía, comunicaciones, informática, y en general en el incremento de la calidad de vida.

"La tecnologia fue creada para la comodidad del hombre, porque si no hubieran tenido esa necesidad no hubieran tratado de inventar nada, está asociado a tratar de mejorar la calidad de vida del hombre" (Grupo de Ingeniería, UPLA).

"Yo creo que el ser humano no podria vivir sin tecnologia, quizás antes no la llamaban así... el ser humano anda buscando cosas... siempre quiere mejorar lo que tiene, creo que es imposible que viva sin tecnologia, sin herramientas, para tener una mejor calidad de vida" (Grupo de Ingeniería, USACH).
En su lado negativo, los discursos tienden a coincidir en la amenaza que representa la informática para la sociabilidad de las personas, en términos de la deshumanización de los actos comunicativos. También se percibe riesgo por el "alejamiento de la naturaleza", la "incertidumbre del futuro" y lo peligroso que es el tema de la "clonación de seres humanos", que se relaciona con valores y creencias religiosas. Asi mismo se vincula al desarrollo científico-tecnológico con el desarrollo armamentista.

"Tenemos mucha contaminación y como que nadie se preocupa de eso, todos están felices porque están ganado más plata, pero nadie se preocupa de si estamos destruyendo el planeta"... (Grupo de Ingeniería, UPLA).

"Tecnologia, bueno, la tecnología yo creo que se va creando a medida que se necesite algo, o sea, generalmente la tecnología se desarrolla en las guerras, es lo que uno ve, porque sí invierten en investigaciones para eso"... (Grupo de Ingeniería, USACH).

En general, se concuerda que el desarrollo científico-tecnológico no es intrínsecamente malo, sino más bien neutro. El empleo y la evaluación de los efectos de este desarrollo, sin tomar conciencia sobre los valores y el medio ambiente que le involucra, es lo que lo hace peligroso e incierto a este desarrollo.

Se opina que tanto las oportunidades como los riesgos implícitos en el desarrollo de nuevas ciencias y tecnologías, dependen, a fin de cuentas, de la valoración de cada uno y de cómo la sociedad considere bueno o malo aquello que crea, innove, desarrolle o produce. Aunque no hay clara sistematicidad en el discurso se reconoce un condicionamiento ideológico y ético del tema.

"También depende de la visión, se supone que todos los avances tecnológicos son para favorecer la condición del ser humano, o sea, van en pro de que sea más cómoda la vida, de que tus preocupaciones sean no trabajar más duro y más duro en el ser humano en general, sino que tu vida 
Revista CUHSO volumen $16 \mathrm{~N}^{\circ} 2$

sea preocuparte de cosas más importantes para ti, de tu familia, de tus valores, etc. La tecnología hoy dia se utiliza para favorecer a algunos, y otros siguen teniendo que trabajar, y se genera cesantía y trabajos que duran menos tiempo, que son mal remunerados, pero yo creo que va más no en el desarrollo tecnológico por sí solo, sino que en cómo se oriente el desarrollo tecnológico" (Grupo de Ingeniería, USACH).

Se apela, en última instancia, a la formación valórica que cada individuo recibe en su núcleo familiar.

"... creo que en si la tecnología no tiene... o sea, el desarrollo de tecnología... el responsable no es la tecnología ni el desarrollo de ella en sí, sino las aplicaciones de las personas y los valores que tiene inculcados cada persona y cómo uno aplica esas tecnologias".

"...si se aplicaran las tecnologías que hoy existen y están desarrolladas, no tendría por qué haber hambre ni tampoco gente pasar frío... Pero esas tecnologías no se aplican a ciertos pueblos por... valores económicos, individualistas... Así que, y es ese es el problema, el problema fundamental está en los valores de la sociedad que cómo aplica la tecnología y no en sí la tecnología”. (Grupo de Ingenieria, UPLA).

\section{Conclusiones del discurso de los estudiantes}

En sintesis, podemos recoger algunas de las ideas más sobresalientes del discurso de los estudiantes para elaborar las siguientes conclusiones y reflexiones de orden más general:

- Existe conciencia de la necesariedad de la ciencia y, todavía más, de lo imprescindible de la tecnología en la sociedad contemporánea. Se puede afirmar que estos estudiantes, en su mayoría nacidos en la década del ochenta, son "hijos de su tiempo". Nacieron bajo el impulso de las grandes tranformaciones, de las nuevas tecnologías de la computación y de la comunicación, y en su mayoría han sido socializados bajo sus influjos.
- Se reafirma la visión positiva de las cien. cias y la tecnologías pero matizadas por la clara conciencia de sus riesgos. Los estudiantes de humanidades manifiestan una mayor perocupación crítica. Es interesante destacar que esta "perplejidad relativa" dice relación, en el discurso, con la incertidumbre que subyace y se cierne sobre el horizonte cultural de estos estudiantes.

- Las nuevas corrientes de las epistemologías comienzan a permear el sentido común científico de los estudiantes de hoy en cuanto al reconocimiento, aunque no formulado sistemáticamente, ni desarrollado en forma homogénea, de la complejidad de las ciencias, de las distinciones entre ciencias duras y blandas y sus fronteras movedizas, y en la superación del positivismo clásico.

- A pesar de lo anteriormente mencionado, subsiste -en forma matizada- una jerarquización de las ciencias ( $\mathrm{y}$ que supone una desvalorizacion relativa de las ciencias humanas) por parte del discurso de estudiantes de tecnologías. Hay un esfuerzo de reconocimiento, pero parece susbsistir una visión bajo la cual la mayor jerarquía en el quehacer de las ciencias se le otorga a las ciencias exactas y naturales.

- Por su parte, el discurso de los estudiantes de humanidades es más crítico. El enfoque crítico de estos dice relación con una mirada integral y contextual en la cual el quehacer especializado de las ciencias y la tecnología deben inscribirse en un conjunto de preguntas más fundamentales que lo evalúan en términos de su impacto en el destino global de la sociedad: son preguntas por el sentido del desarrollo humano, la equidad y el desarrollo sustentable.

- El análisis crítico de parte del discurso de los estudiantes de tecnologías - cosa que resulta interesante notar-, a pesar de que no tiene la conntación de una crítica global, se orienta, revelando una racionalidad pragmática, hacia la preocupación por el empleo de la tecnología. Y este está centrado en el efecto positivo o negativo sobre el desarrollo humano de las nuevas tecnologías de información y comunica- 
ción en relación a su impacto en los procesos de sociabilidad. El discurso crítico de ingenieros destaca ámbitos relevantes de las ciencias y tecnologías que el discurso de los humanistas parece olvidar: las biotecnologías, estas nuevas tecnologías informáticas y comunicacionales, y cuestiones relativas a la energía.

- En último término, el discurso de los humanistas remite a un análisis teleológico de la tecnología, en cambio, el discurso de los estudiantes de tecnologías - estableciendo la neutralidad intrinseca de ellas- se centra en un análisis extrínseco y contextual. En todo caso, más allá de la forma cómo se concibe a la ciencia y la tecnologia, la critica desde un codigo ecológico -fuertemente alimentado por las preocupaciones medioambientales es un dato común y de mucha gravitación en ambas visiones.

- En ambos discursos los valores resultan importantes. Por una parte, parece percibirse un reconocimiento de la diversidad cultural como cuestión que en el día de hoy afecta la visión de las ciencias y la tecnología. Asimismo parece central un cuestionamiento ético que se ve corroborado en los datos de la encuesta cuantitativa ${ }^{5}$.

- En el discurso de los estudiantes de humanidades, como resultaría más lógico esperar, al reflexionar sobre ciencia y tecnología surgen temáticas de relevancia cultural que están en el debate contemporáneo y que dicen relación con los desafíos de las identidades en el marco de los procesos de globalización

- En ambos discursos emerge la importancia de la educación en términos de su papel socializador y formador de personas en relación a la ciencia y la tecnología. Se entrega una reflexión crítica cuando se analiza sus posibles desviaciones $\mathrm{y} / \mathrm{o}$ su contribución a deformar conceptos sobre la ciencia y la tecnología. En particular se critica una visión ingenua y acrítica que la educación pueda estar entragando a los alumnos. La educación, en definitiva, presenta un espacio de oportunidad, pero también de desafíos.

En suma, no hay tal abismo entre las "dos culturas"; al menos en el discurso que hemos analizado. Hay ciertamente diferencias y matices. Claro que en un aspecto decisivo hay una diferencia teórica, con connotaciones éticas. La naturaleza misma de la tecnología: para los estudiantes de humanidades se trata de una práctica social, humana y por ende sometida a condicionamientos históricos y culturales reconocibles; por lo mismo el análisis ético de ella deberia asumir también la naturaleza y el tipo de tecnología: hay también una valoración de la orientación y de los fines hacia los cuales conduce este desarrollo. Para los estudiantes de ingenerías, en cambio, se trata de una práctica "técnica" sometida a leyes con autonomia propia y, por tanto, con posibilidad de establecer una "neutralidad valorativa" sobre dicha práctica: la tecnología es una herramienta neutra, depende de la valoración y uso que se haga, de sus contextos, de si ella es bien o mal empleada para beneficio o destruccción de la humanidad.

A la luz de estos discursos de estudiantes universitarios es posible afirmar que existirian las condiciones para un diálogo fructífero entre las ciencias -tecnologías y humanidades- en torno a las definiciones de ciencias y tecnologías y sus aportes e impactos en el mundo contemporáneo. El discurso universitario parece estar estructurado en torno de códigos de interpretación de realidad no unívocos. Se trata de discursos que remiten

En el estudio de 2005 un relevante 91\% de los estudiantes afirman que están de acuerdo con la frase: "El adecuado manejo ético y político de la invención científica permite grandes logros y avances para la humanidad". De ellos: un $44 \%$ declara estar "plenamente de acuerdo" y un 47\% afirma estar "de acuerdo" con ella. En el estudio de 2008 un $85 \%$ afirman estar de acuerdo con la misma frase ( $39 \%$ plenamente de acuerdo y $46 \%$ de acuerdo). Por otra parte, un $89 \%$ de los estudiantes, casi nueve sobre diez en el estudio de 2005 y $85 \%$ en el estudio de 2008 , con mayor énfasis (acuerdo pleno) o no (simple acuerdo) declaran que la ética es una actitud y una reflexión que se está tornando progresivamente necesaria frente al avance de los progresos tecnocientificos de punta en las áreas de las biotecnologias (incluyendo la bioinformática) y la robótica. Cfr. Parker (2008a) y Parker (2008c). 
a semantizaciones un poco más complejas y críticas, al menos reflexivas en el sentido pospositivista: lo que posibilita reconocimientos mutuos entre las humanidades (y sus lógicas interpretativas) y las ciencias -tecnologías (y su lógica-experimental) aun cuando subsisten, por cierto, sus diferenciaciones. El punto de encuentro parece estar en la necesidad de una evaluación ética y ecológica tanto de las ciencias (naturales o humanas) como de las tecnologías.

\section{Reflexiones postestudiantiles}

En el mundo actual efectivamente hay caminos de búsqueda de replanteo de la relación entre el pensamiento científico (incluido el pensamiento tecnológico) y las humanidades (incluidas las reflexiones estético-culturales) (Ramírez, 2005). Algunas corrientes de pensamiento epistemológico recientes como la hermeneútica, las posturas postkuhnianas y las teorias del pluralismo cultural y multicultralismo proporcionan las bases para sustentar, con cierto optimismo, un encuentro en la "multidisciplina" que no niega la especificidad disciplinar pero que ciertamente posibilita un trabajo conjunto entre disciplnas en una sociedad del conocimiento que cada día más requiere de este tipo de aproximaciones para resolver los problemas complejos que le desafían.

Zygmunt Bauman (2005), en su excelente caracterización de los cambios culturales de la actual fase histórica, nos habla de la "modernidad líquida". Estamos pasando de la modernidad pesada a la modernidad liviana nos dice, de la era del hardware a la era del software.

De la sociedad industrial, la era de las grandes máquinas y edificios, a la era de las computadoras, la era de los chips y las nano y biotecnologías. La modernidad pesada fue la época de la conquista territorial: la riqueza y el poder estaban arraigados en tierra, eran pesados, y de movimientos torpes, dependían del tamaño y de la calidad del hardware. El acero y el concreto eran sus materiales. En la nueva sociedad estamos en la era del poder de la información.

Pensamos que las ciencias naturales $y$, sobre todo, cierta forma reductivista, mecanicista y positivista de ellas se acomodó de forma bastante adecuada a la modernidad pesada que es la de la época industrial. Así el capitalismo en sus orígenes, tanto como el socialismo real, se valieron de grandes impulsos dados por el desarrollo científico-tecnológico, pero quizás descuidando aspectos humanos y éticos. Siempre hubo científicos que abogaron por principios y debemos recordar el pacifismo que caracterizó por ejemplo a Einstein. Pero, claro, dichas posturas se desarrollaron luego del holocausto y de la bomba atómica.

Ahora esta modernidad líquida plantea nuevas interrogantes, nuevos desafíos y nuevas perspectivas a las ciencias. Las dimensiones de la economía de la información, con su mayor flexibilidad (en los proceso de producción y servicios; en el mercado de trabajo y el mercado financiero), la mayor injerencia de las subjetividades, la menor relevancia de las estructuras, la mayor influencia de lo estético y lo simbólico, y la creciente complejidad de una sociedad del conocimiento basada en las nuevas tecnologías de la información y de la comunicación, transforman las formas de producción del conocimiento, y plantean desafios inéditos a las ciencias mucho mayores incluso que aquellos que planteó la revolución de la física quántica y la teoría de la relatividad a la mecánica newtoniana.

El punto más promisorio de la modernidad pesada, con su inquebrantable confianza en la planificación (social) y en la predictibiliad (científica), se ha ido. También se ha superado el paradigma de la causación simple y su unilinearidad y ahora se reconoce que la realidad está sometida a relaciones complejas donde incluso hay patrones cambiantes de impredictibilidad. La popularización de la teoria del caos en las ultimas décadas del siglo XX es toda una metáfora del cambio que se presencia. Por ello, el reconocimiento de la complejida creciente supone a su vez, en el intento de reducción de la complejidad, que es el esfuerzo de las ciencias, un espacio muy interesante e importante de interfases entre las ciencias naturales, las humanidades y las ciencias sociales (Helga Nowotny, 2005).

Desde otro enfoque, el desarrollo de los análisis que ponen al lenguaje en el cen- 
tro de la reflexión sobre la realidad social, que arranca de la semántica y del estructuralismo, tanto como de la filosofia analitica del siglo $X X, y$ que han sido fuente de reflexión teórica para tantos autores de la ciencia natural, la epistemología y las ciencias sociales, plantean un reconocimiento central de condicionamientos que vienen a cuestionar la construcción de las ciencias sobre la base de lógicas exteriorizadas las unas de las otras y totalmente autónomas, y se dejan tendidos puentes entre las diversas formas cientificas de abordar las realidades naturales y sociales.

Como afirma Rorty:

"Se operó un cambio en la filosofía analitica cuando los filósofos comenzaron a leer a Wittgenstein y sus "investigaciones filosóficas" de la mano con Kuhn y su "Estructura de las Revoluciones cientificas".

El resultado de esta colaboración ha sido el borrar las fronteras entre las ciencias y las humanidades y la tentativa de hacer de la controversia de Snow (las dos culturas) tan pintoresca como el debate del siglo XIX sobre la edad de la tierra" (Rorty, 2000 , p. 23).

En este marco hay quienes comienzan a defender a la clásica forma de hacer ciencia del "relativismo" que las amenazaría. La propia sociología de la ciencia ha estado en un debate acerca de las implicancias de las tradiciones normativas y naturalistas (ver Pels, 1996), debate que no viene al caso desarrollar aquí, pero que es ilustrativo de la complejidad espistemológica del problema.

Por ello, no resulta extraño que la llamada guerra de la ciencia (Cohen, 2001) ha sido en este último tiempo -sobre todo en el mundo anglosajón- el debate entre los estudiosos de la ciencia y los autoproclamados defensores de esta.

Los defensores reclaman que la autoridad de la ciencia ha sido minada por los estudiosos de la ciencia (filósofos, historiadores, sociólogos, antropólogos, espistemólogos) y especialmente por los argumentos constructivistas que afirman que la ciencia no es más que un "constructo" humano.
Los estudiosos de la ciencia proponen la noción de que la importancia de la ciencia en, por y para la sociedad, necesita que se dé cuenta de manera más compleja de lo que realmente es la ciencia. Uno de los asuntos claves es que se defina con mayor precisión lo que se entiende por "constructo social", y si este va antecedido de la frase "nada más que".

El asunto no es fácil y las posibles respuestas provendrán de los paradigmas y perspectivas desde las cuales se analice el problema.

Cierto es que en las formas de produccción de las ciencias contemporáneas, la vieja división del trabajo intelectual entre ciencias naturales y ciencias humanas, como compartimentos estancos que no se hablan, está siendo desafiada e incluso superada. A cada momento emergen áreas de realidad que inesperademente cuestionan los márgenes de los dominios, órdenes y formas del conocimiento. Esta reducción de la naturaleza absolutamente diferenciadora de las fronteras de realidades no deja de ser problemática. Las interfases de las ciencias - y que posibilitan una creciente cooperación inter, multi y transdiciplinaria- desafian también las definiciones clásicas. Las fronteras y los sentidos que se les asocian están cambiando al mismo tiempo: entre lo que significa ser humano y no humano, entre agentes y objetos, y lo que puede ser tratado como objeto, entre el yo y el otro, entre la cooperación humana y el conflicto y cómo se construirán las futuras identidades. "Las fronteras podrían ser reconstruidas en el marco de las ciencias pero conllevan un sentido y este es creado por la sociedad. Los sistemas complejos en evolución no pueden dejarse solamente a la cien. cia, dado que están entretejidos con la complejidad de los sistemas sociales. Involucran a agentes humanos y a las cosas, a la ciencia y a la sociedad en nuevas configuaciones. Las novedades cientificas necesitan ser leidas y comprendidas de una manera socialmente significativa, para ser apropiados" (Nowotny, 2005: 28).

Lo cierto es que las fronteras entre las disciplinas han comenzado ha desdibujarse en todos los campos y el reconocimiento de la 
complejidad de los fenómenos - naturales y/o sociales- está formando parte ya de la cultura científica del siglo XXI (Parker, 2008b).

Por cierto, nos referimos a la auténtica apreciación acerca de la complejidad fenoménica y no a aquella moda postmodernista que, de manera poco rigurosa, termina por confundir las realidades analíticamente distinguibles en conceptos imprecisos y rimbombantes como "hibridación de perspectivas" o "juego de lenguajes que conllevan la fragmentación".

Que nos estemos abriendo a la posibilidad de la porosidad de márgenes no significa, de ninguna manera, que habremos de rendirnos del esfuerzo metódico por sostener los principios disciplinarios; pero ciertamente necesitamos reconocer los nuevos modos de producir conocimiento en este siglo que se inicia y donde la inter y multidisciplinariedad tienen un lugar destacado.

Un aspecto relevante de esta nueva cultura cientifica que emerge desde el inicio de la acentuación de la globalización hacia fines de la década de los 70 y que se ve reforzada por el término de la Guerra Fría y sus consecuencias para la internacionalización de la ciencia-técnica y las nuevas formas de su producción, es que se ha superado el paradigma antropocéntrico-racionalista que sustentaba una concepción prometeica de la ciencia.

En este sentido es posible sugerir un cambio en los términos de nuestro problema dado que es posible hablar de que no hay dos polos opuestos, ciencia, por un lado, y humanismo, por otro.

Como afirma Morin (2005), es posible incluir a la ciencia como una parte muy relevante del humanismo occidental. Claro que este humanismo - continúa Morin- tiene dos rostros, uno dominante y otro fraternal.

El primer rostro del humanismo -desde el Renacimiento- coloca al hombre en lugar de Dios, le hace el único sujeto del universo y le da como tarea conquistar el mundo. Es la misión que Descartes concede a la ciencia: hacer del hombre el dueño y señor de la naturaleza. Retomado por Bufón, Marx y tantos otros, el mensaje cartesiano de la omnipotencia prometeica cae hecho pedazos recién en las últimas décadas del siglo XX.

Advertimos ahora que este tipo de dominio de la naturaleza, que de suyo no admite control, lleva a la degradación de la biosfera y por consiguiente de la vida y sociedad humana. Hay aquí una suerte de tendencia suicida.

Ahora adquirimos conciencia de la contingencia y de la pequeñez del planeta y del hombre en el universo, en este universo que se expande y que a cada instante nos sorprende con fenómenos que creíamos conocidos pero que la astrofísica nos revela misteriosos y maravillosos.

$Y$ he aqui el segundo rostro del humanismo: el que se reconoce humilde, el que redescubre la dignidad humana y el valor de todo lo viviente en la faz de la tierra. Un humanismo que también reconoce sus errores, sus barbaries, sus retrocesos... un humanismo que reconoce la diversidad de culturas, experiencias, inteligencias, racionalidades. "En toda sociedad coexisten un pensamiento racional, técnico y práctico, y un pensamiento mágico, mítico y simbólico" (Morin 2005: 49). Recordarlo nos parece indispensable para reubicar la nueva epistemología que ubica a las ciencias como una forma de conocimiento, valioso y válido, pero no exclusivo, ni menos apodíctico y monopólico.

La superación del positivismo clásico y también del positivismo renovado, de ese racionalismo determinista y cientificista o logocéntrico, no supone de ninguna manera admitir como válidas las afirmaciones del relativismo, sino significa ubicar un ámbito de competencia y validez para el quehacer científico, una legitimidad para el método y sus resultados, con sus grandes aportes, pero en el marco del reconocimiento de sus propios límites y condiciones sociales y epocales.

Por lo mismo, que las ciencias consideren de manera tan relevante a la ética en nuestros días, no debe ni puede convertirse en una "moda" intelectual más, sino un imperativo propio de los nuevos paradigmas que alientan al desarrollo cientifico desde 
sus inspiraciones más profundas por un conocimiento verdadero, abierto a la crítica y a la novedad. El dogmatismo es tan contrario a la ciencia como lo es la autocomplacencia de quien, poseedor de una parte de la verdad, se cree en posesión de un poder omnímodo para imponer su dominio sobre los otros.

Tampoco se trata del retorno al humanismo clásico, a aquel que tuvo sus fundamentos en una ontología y metafísica premoderna fatalmente desfasada con los nuevos conocimientos que nos entregan, desde la antropología contemporánea, hasta la biología, la fisicoquímica y la física cuántica actual.

Por ello, el replanteo de las "dos culturas" que ahora comienzan a dialogar, a interactuar, e incluso a trabajar juntas, no solo supone superar el paradigma cerrado de la ciencia natural, sino que también supone un enorme desafío a las humanidades y a la ciencia social contemporánea. $Y$ en este sentido hay, por cierto, variados y diversos campos en los cuales las ciencias sociales y humanas tienen que abrirse al aporte de los avances de las ciencias actuales: biotecnologías, genoma, nanotecnologías, nuevas biologías, fisicoquímica subatómica, astrofísica, etc.

Finalmente una cuestión inspirada en el pensamiento de nuestros estudiantes universitarios actuales: la nueva forma de concebir a la ciencia supone el análisis simultáneo de sus responsabilidades éticas: a) hacia las personas y grupos humanos, tanto como b) hacia la biosfera. En ambos casos hay puntos de confluencia entre ciencias naturales y ciencias humanas.

En términos de Hans Jonas (1995), el porvenir en la tierra depende, no de la esperanza en una utopía lejana, sino en el ejercicio de una libertad responsable y en un contexto -como la sociedad tecnológica-caracterizado por el enorme poder humano sobre la naturaleza, desconocido hasta nuestra época. Se trata de una exigencia ética que toma en consideración la condición de posibilidad de la vida, del entorno biótico, que estaría dada precisamente por la asunción de una responsabilidad antropocéntrica, en sintonía profunda con la naturaleza, lo que garantizaria, por lo demás, el porvenir del hombre y de su entono.

Respecto al pensamiento holístico es necesario recordar que este no es solo un producto intelectual del pensamiento sistémico presente en las ciencias del siglo XX. Es también y quizás de manera más decisiva, producto de una toma de conciencia ecológica de la humanidad de fines del siglo pasado.

Edgar Morin afirma que:

"Una idea ha comenzado a despuntar en las últimas décadas del siglo $X X$, aunque sus orígenes son antiguos: la de una nave espacial, la tierra, donde navega la humanidad.

Esta nave es propulsada hoy por cuatro motores: ciencias, técnica, economia y ganancias, y esos motores no están bien controlados. No me inscribo en un pensamiento binario y no digo que la ciencia es mala, al contrario, pero digo que ha desarrollado poderes de destrucción inauditos $e$ incontrolados. El desarrollo técnicoeconómico actual produce la degradación de la biosfera, que a su vez arrastra la degradación de la civilización humana..." (Morin, 2005: 72).

Por cierto, la ética es ahora considerada en el ejerció de las ciencias. Peĩo ella no debe ser considerada uni factor exógeno, un complemento externo que solo determina condiciones de viabilidad y regulación a los proyectos de investigación; la ética es también un componente hoy del mismo quehacer de la ciencia: la bioét ica no es solo una disciplina de especialistas, sino una exigencia del ejercicio responsable de la ciencia a la luz de los graves desafíos de un mundo que reclama nuevas posibilidades para un desarrollo humano integral y sustent able.

En suma, no es posible pensar de manera ingenua $y / 0$ voluntarista que se hayan borrado las distinciones entre ciencias naturales y humanismo. Es más, hay que pensar que no existen "dos culturas" enfrentadas, sino muchas "culturas" en situación de coexistencia/competencia y/o colaboración: la cultura científica "dura" (fisico-químicaexperimental) y exacta (ciencias formales); 
la cultura tecnológica (ciencias aplicadas y ciencias innovativas y propiamente tecnológicas); la cultura de las ciencias sociales (que también está atravesada por el debate entre cientificismo y humanismo) y las humanidades (que incluye a las letras, la música y al arte), entre otras.

Las fronteras se hacen borrosas y porosas pero siguen existiendo y son necesarias. Claro que el desafío de los modos de hacer ciencia y de producción de conocimientos en este siglo nos lleva a reconocer espacios cada vez más significativos de intrersecciones, interfases, intercambios y cruces transversales; sobre la base de la asunción de los alcances y límites de los respectivos campos de competencia y validez. A fin de cuentas existe en todo ser humano el potencial del científico y del artista, y en realidad somos algo de ambos (Truman, 1988). Pero la especialización, necesaria en este época centrada en el crecimiento, la economía y los mercados, podría hacernos una mala jugada cuando nos encierra en estancos que ciegan con pesadas anteojeras nuestras visiones.

Las diversas culturas científico-humanistas tienen mucho de qué beneficiarse de un programa de colaboración, pero a condición de no procurar imponer su discurso y su práctica de manera hegemónica. Con todo, es riecesario ir nîás allá de la clásica división de las "dos culturas". asentada en un paradigma ya superado (pero tiodavia vigente en muchos discursos y prácticas). La preeminencia de un enfoque humanista actual que supere la racionalidad instrumental y meramente funcional, aun asumiendo las características diferenciadoras de las actividades y praxis de las disciplinas humanistas respecto de las científicas y estas respecto de las tecnológicas-ingenieriles, pasa por asumir un enfoque sobre el sentido de esas acciones. El papel de la educación (Bugliarello, 2003) en todo esto puede resultar decisivo.

El sentido teleológico de la ciencia no puede agotarse en la pregunta acerca del avance del conocimiento, sin más, dado que este, precisamente en la sociedad del conocimiento, es fuente de poder y las decisiones que se tomen acerca de la direccionalidad de las ciencias no son indiferentes respecto al destino futuro de la humanidad en este planeta.

Los estudiantes universitarios -al menos el tipo de estudiantes cuyo discurso hemos analizado- parecen estar en una buena disposición para emprender un diálogo fructifero entre ciencias naturales y humanas, bajo una triple condición: a) que su formación sea profundizada en términos de los fundamentos epistemológicos y valóricos de sus propias disciplinas; b) que, más allá del voluntarismo, sea reconocida la dinámica objetiva y problemática en las relaciones entre ciencias naturales y ciencias humanas, y c) que la aplicación de la ciencia al mundo de la vida sea efectuada sobre la base de criterios técnico-prácticos, pero asumiendo sus impactos propios, por lo que no debe descuidarse las permanentes evaluaciones éticas de dichas prácticas.

\section{Referencias}

ZYGMUNT BAUMAN (2005), Modernidad Liquida, Fondo de Cultura Eonómica, Buenos Aires.

GEORGE BUGLIARELlO (2003), "A New Trivium and Quadrivium", Bulletin of Science Technology Society, vol. 23, pp. 106-113.

BENJAMIN R. COHEN (2001), "That Have Exemplified Cross-Disciplinary Tension On the Historical Relationship Between the Sciences and the Humanities: A Look at Popular Debates", Bulletin of Science Technology Society vol. 21, pp, $283-295$.

ANDREW FEENBERG (1999), Questioning Technology, Routledge, New York.

JURGEN HABERMAS (1973), La Technique et la science comme "ideologie", Gallimard, Mesnil-sur-l'Estée, 1973.

ALDOUS HUXLEY (1979), Literatura y Ciencia, trad. Rubén Masera, $2^{a}$. ed., Sudamericana, Buenos Aires.

HANS JONAS (1995) El principio de responsabilidad: ensayo de una ética para la civilización tecnológica, Barcelona, Editorial Herder.

TOMAS KUHN (2005), La estructura de las revoluciones cientificas, Fondo de Cultura Económica, Madrid. 
UNIVERSIDAD CATÓLICA DE TEMUCO

EDGAR MORIN (2005), Breve historia de la barbarie en Occidente, Paidós, Buenos Aires.

DIETER NOHLEN (2006), "Método Comparativo", en Diccionario de Ciencia Política: Teorías, métodos, conceptos, dos tomos, Ciudad de México: Porrúa, 2006, En http://www. nohlen.uni-hd.de/es/doc/diccionario_metodocomparativo.pdf

HELGA NOWOTNY (2005), "The Increase of Complexity and its Reduction: Emergent Interfaces between the Natural Sciences, Humanities and Social Sciences", Theory, Culture \& Society, vol. 22(5), pp. 15-31.

CRISTIÁN PARKER G. (2008a) "Science and Technology in Undergraduate Students Worldview, Shaped by Globalization: The Chilean Case", Perspectives on Global Development and Technology, vol. 7, $\mathrm{N}^{\circ} 1$, pp. 67-90.

CRISTIÁN PARKER G. (2008b) "Por una nueva cultura cientifica: más alla de las dos culturas", Revista Estudios Avanzados, vol. 6, N¹0, pp. 7-24.

CRISTIÁN PARKER G. (2008c) "Alfabetismo científico y conciencia crítica en estudiantes universitarios chilenos", en Roberto Leher (comp), Por uma reforma radical das Universidades Latino-Americanas, CLACSO, Buenos Aires.

DICK PELS (1996), "Karl Mannheim and the Sociology of Scientific Knowledge: Toward a New Agenda", Sociological Theory, vol. 14, issue 1, Mar. pp. 30-48.

MARIO TEODORO RAMIREZ (2005), "Ciencias y humanidades. De la oposición a la complementación", II Congreso Iberamericano de Filosofía de la Ciencia y la Tecnologia, Ciencia, Tecnologia y Ciudadanía en el Siglo XXI, Universidad de La Laguna, Tenerife, España, 26 al 30 septiembre 2005 .

RICHARD RORTY. (2000), "Being that can be understood is language: Richard Rorty on H.-G. Gadamer", London Review of Books, March 16, pp. 23-25.

CHARLES PERCY SNOW (1993), The two cultures, Cambridge: Cambridge University Press (Original 1959).

A. TRUMAN SCHWARTZ (1988), "Science: the Greatest of the Humanities?", Bulletin of Science Technology Society, vol. 8; pp. 167-171. 